Pacific Journal of Mathematics

A DIRICHLET-JORDAN THEOREM FOR DUAL 


\title{
A DIRICHLET-JORDAN THEOREM FOR DUAL TRIGONOMETRIC SERIES
}

\author{
ROBERT B. KELMAN
}

\begin{abstract}
An analog of the Dirichlet-Jordan theorem and a uniqueness theorem are established for dual trigonometric series equations when the right hand sides of the dual equations are given functions of bounded variation. In the usual fashion there are two series in these equations one of which has coefficients, say, $\left\{\left.j\right|_{n} n\right\}$ or $\left\{j_{n} / n-1 / 2\right\}$, and the other coefficients $\left\{j_{n}\right\}$. In the first series we establish ordinary convergence and in the second Abel-Poisson convergence. In general $j_{n} \neq o(1)$ and the second series does not converge in the ordinary sense on any set of positive measure. $A$ best possible estimate on growth conditions for $\left\{j_{n}\right\}$ needed for uniqueness is given. In the proof a mixed boundary value problem of potential theory is associated with the dual series. Conformal mapping replaces this potential problem with one in which a Dirichlet boundary condition can be associated with the dual series. Analysis of this new problem provides the denouement.
\end{abstract}

1.0. Problem statement. Perhaps the most important theorem in the application of Fourier series is the celebrated result of Dirichlet and Jordan [4, p. 114; 26, p. 57] describing the behavior of trigonometric expansions of functions of bounded variation. I establish here an analog of this theorem and the uniqueness of the expansions for the four dual trigonometric equations given below which are the canonical forms to which all classical dual trigonometric series can be reduced by elementary transformations [19, p. 150]. Let $\mathfrak{p}$ denote the interval $0<x<\pi$ and $b$ be a fixed point in $\mathfrak{p}$. Let $\mathfrak{l}$ denote the interval $0<x<b$ and $\mathfrak{x}$ the interval $b<x<\pi$. Let $f(x)$ and $g(x)$ be functions of bounded variation for which we use the notation $\hat{f}(x)=(f(x+0)+f(x-0)) / 2$. The dual trigonometric series to be studied are:

$$
\begin{gathered}
\lim _{r=1-0} \sum_{n=1}^{\infty}\left(\frac{j_{n}}{n-1 / 2} \cos (n-1 / 2) x\right) r^{n}=\hat{f}(x), x \in \mathfrak{l}, \\
\lim _{r=1-0} \sum_{n=1}^{\infty}\left(j_{n} \cos (n-1 / 2) x\right) r^{n}=\hat{g}(x), x \in \mathfrak{x} ; \\
\lim _{r=1-0} \sum_{n=1}^{\infty}\left(\frac{j_{n}}{n-1 / 2} \sin (n-1 / 2) x\right) r^{n}=\hat{f}(x), x \in \mathfrak{I},
\end{gathered}
$$




$$
\begin{gathered}
\lim _{r=1-0} \sum_{n=1}^{\infty}\left(j_{n} \sin (n-1 / 2) x\right) r^{n}=\hat{g}(x), x \in \mathfrak{r} ; \\
\lim _{r=1-0} \sum_{n=1}^{\infty}\left(\frac{j_{n}}{n} \sin n x\right) r^{n}=\hat{f}(x), x \in \mathfrak{l}, \\
\lim _{r=1-0} \sum_{n=1}^{\infty}\left(j_{n} \sin n x\right) r^{n}=\hat{g}(x), x \in \mathfrak{r} ; \\
j_{1}+\lim _{r=1-0} \sum_{n=1}^{\infty}\left(\frac{j_{n+1}}{n} \cos n x\right) r^{n}=\hat{f}(x), x \in \mathfrak{l},
\end{gathered}
$$$$
\lim _{r=1-0} \sum_{n=1}^{\infty}\left(j_{n+1} \cos n x\right) r^{n}=\hat{g}(x), x \in \mathfrak{x} \text {. }
$$

1.1. Background. These dual series have primarily been examined in connection with applications, especially in mechanical engineering as explained in $[19]$ (see $[1 ; 2 ; 9 ; 12 ; 20 ; 16]$ and references $[6 ; 8]$ in $[10]$ for more recent applications). Understandably this has led to the development of formal answers and special methods with little information on the limitations needed to insure their validity, cf., $[19 ; 4 ; 7 ; 10$; $24]$ and references $[1 ; 2 ; 9-17]$ in [10]. This paper was motivated by the desire to present a unified approach to these equations and to answer basic mathematical questions of existence, uniqueness, and behavior raised earlier. The need for this was made more urgent by Srivastav's interesting formal construction [21] showing eq. (4A-B) does not have a unique solution and the discussion that has occurred for some solutions e.g., reference [1] in [10] and Math. Rev. 37 (1969), \#5632.

Shepherd [18] established rigorously the existence of a solution to $(3 \mathrm{~A}-\mathrm{B})$ with $b=\pi / 2, f=\sin m x$, and $g=-m \sin m x$. His procedure requires an explicit solution of an infinite vector equation $A p=q$ where $p$ and $q$ are infinite column vectors and $A_{m n}=(m+n-1 / 2)^{-1}$ (more recent applications of this method are found in $[8 ; 6]$ ). By extending the method along lines suggested in [18], one can obtain a special case of the results given in Theorem 3 for eq. (3A-B), but further generalizations appear difficult because of the explicit quantities involved in the proof and the fact that the inverse of $A$ is not unique $[14 ; 22]$. In [23] a general formulation of both dual integral equations and dual series was given, but the results are formal and applications to specific series have not been forthcoming. In [11] we established an existence and uniqueness theory for dual orthogonal equations in Hilbert space applicable to dual series associated with potential problems with mixed boundary conditions of the second and third kind, cf. [5], but as pointed out in [11] this technique breaks down for the series studied here in which one of the mixed conditions is a 
Dirichlet condition.

1.2. Outline and comments. The idea of the proof is this. We associate with each dual trigonometric series a mixed boundary value problem in a semi-infinite strip which we conformally map onto a rectangle to obtain a separated variable solution, since in this form we can use the full armamenatarium of the theory of Fourier series to directly analyse the solution. The rectangle is mapped back onto the original domain in such a way that the dual series can now, in a certain sense, be associated with a Dirichlet condition-an association which is the denouement. To make the required connections between solutions requires uniqueness theorems which we are able to present in a simple manner by means of Wolf's reflection principle [25].

It might occur to the reader that in $\S 2.0$ it would be easier to map the above mentioned strip onto a half-plane and obtain a KeldyshSedova problem. However, this would require more restrictive continuity assumptions on $f$ and $g[13, \mathrm{p} .347]$ and, more importantly, would not permit use of the theory of Fourier series in so direct a fashion as can be done with a separated variable solution in a rectangle.

The gist of the paper can be obtained by reading Theorems 1-3 whose statements involve little technical detail. Roughly, our most important result is that each of the eqs. (iA-B) has one, and only one, solution such that

$$
\sum_{n=1}^{N}\left|j_{n}\right|=o\left(N^{3 / 2}\right)
$$

and for establishing uniqueness this estimate is best possible. As shown in Theorem 3 a general theory of dual series cannot limit itself, as has been the case previously, to ordinary summation, since in general the series in (iB) diverge almost every where in the ordinary sense even for very smooth functions $f$ and $g$, e.g., $f \equiv 1$ and $g \equiv 0$ in eq. $(2 \mathrm{~A}-\mathrm{B})$. There is merit, I believe, in pointing this out.

1.3. Notation. Standard notation for Fourier series is used [4;26]. $\quad \bar{R}$ will denote the closure of $R$. Integrals and measure are in the sense of Lebesgue. In the set forming symbol, say $\{z: A(z)\}$, we often suppress the bound variable when the meaning is clear and write $\{A(z)\}$. Abel-Poisson summability will be called summability $A$. We denote by $\mathfrak{I}_{0}$ the set of points $\{(x, 0): x \in \mathfrak{l}\}$ with $\mathfrak{r}_{0}$ and $\mathfrak{p}_{0}$ defined similarly. Let $f(x, y)$ be defined in a set $R$ and along some open arc $p$ in the boundary of $R$. We say $f$ is continuous at $\left(x_{0}, y_{0}\right) \in p$ (adding 'relative to $R$ ' if needed for clarity) if for each sequence $\left\{\left(x_{n}, y_{n}\right)\right\}$ in $R \cup P$ for which $\left(x_{n}, y_{n}\right) \rightarrow\left(x_{0}, y_{0}\right)$, 
one has $f\left(x_{n}, y_{n}\right) \rightarrow f\left(x_{0}, y_{0}\right)$.

2.0. A Dirichlet-Jordan theorem. In our proof we make use of the following theorem which paraphrases results in [25].

THeOREM W. Let $D$ be a domain in the upper half plane which contains an open segment $\mathrm{c}$ of the real axis on its boundary. Each point of c contains a circular neighborhood whose upper half lies in $D$. Let $\phi(x, y)$ be a function harmonic in $D, \phi \rightarrow 0$ as $y \rightarrow+0$ for $x \in \mathfrak{c}$, and $\phi=o\left(y^{-2}\right)$ as $y \rightarrow+0$ uniformly in $x$ for $x \in \mathfrak{c}$. Then $\phi$ can be analytically continued into the domain $D^{*}$ symmetric to $D$ with respect to the real axis. If $\psi$ is conjugate to $\phi$ and $\psi=o\left(y^{-2}\right)$ as $y \rightarrow+0$ uniformly in $x$ for $x \in \mathfrak{c}$, then $F=\psi+i \phi$ can be analytically continued into $D^{*}$.

REMARK 1. This is based on Theorems $C$ and D in [25] in which there is allowed an exceptional subset $T$ of $c$ on which $\phi$ need not tend to zero as $y \rightarrow+0$. If $T$ is not the empty set, it is easy to see that these theorems are in need of modification, vid., Math. Rev. 9(1948), p. 420. Our use is limited to the case in which $T$ is the empty set.

We proceed to our main result.

THEOREM 1. Let $f$ and $g$ be functions of bounded variation on $\overline{\mathfrak{l}}$ and $\overline{\mathfrak{x}}$ respectively. Then there is one, and only one, solution $\left(j_{1}\right.$, $\left.j_{2}, \cdots\right)$ satisfying (5) to each of the dual trigonometric equations (1A-B), $i=1,2,3,4$.

Proof. It is sufficient to give the proof for eq. (1A-B), since the proof for the other three dual equations is practically identical. Let $R_{z}$ be the half strip $\{x \in \mathfrak{p} ; y>0\}$ and $S_{z}$ the subset of $\bar{R}_{z}$ in which $y>0$. We associate with $(1 \mathrm{~A}-\mathrm{B})$ the boundary value problem $P_{z}$ : find a function $T(x, y)$ harmonic in $S_{z}$, bounded in $\bar{R}_{z}$, and satisfying the boundary conditions

$$
\begin{gathered}
T_{x}=0 \text { on }\{x=0 ; y>0\} \text { and } T=0 \text { on }\{x=\pi ; y>0\}, \\
\lim T=\hat{f}(x) \text { as } y \rightarrow+0 \text { for } x \in \mathfrak{l}, \\
\lim T_{y}=\hat{g}(x) \text { as } y \rightarrow+0 \text { for } x \in \mathfrak{r} .
\end{gathered}
$$

REMARK 2. A boundary condition written in the form of (6) implies $T_{x}$ is continuous relative to $R_{z}$ at each point $(0, y), y>0$, whereas (7) only implies for each $x \in \mathfrak{l}$ that $T$ is continuous on the right as function of $y$ at $y=0$. 
In the $w(=u+i v)$-plane let $\lambda$ be the interval $0<u<1$ and $\rho$ the interval $0<v<\kappa$ where $\kappa$ is a positive constant. Let $\lambda_{0}$ be the set $\{(u, 0): u \in \lambda\}, \rho_{0}$ the set $\{(1, v): v \in \rho\}$, and $R_{w}$ the rectangle $\{u \in \lambda$; $v \in \rho\}$. It is well known [13, p. 202] that there exists a function, say $w(z)$, conformally mapping $R_{z}$ onto $R_{w}$ with the correspondences $0 \rightarrow 0, b \rightarrow 1, \pi \rightarrow 1+i \kappa$, and $\infty \rightarrow i \kappa$ for a proper choice of $\kappa$. For future use we note the following properties of $w[13, \mathrm{Ch}$. II $\S \S 11.29$, 3.35, and 3.37]:

(A) $w$ is continuous on $\overline{\mathfrak{x}}_{0}$ and continuously differentiable on $\mathfrak{x}_{0} \cup(0, \pi)$

(B) in the neighborhood of $z=b, w=(z-b)^{1 / 2} t(z)+1$ where $t(z)$ is a function analytic at $z=b$ and $t(b) \neq 0$;

(C) the inverse function $z(w)$ is twice continuously differentiable on $\bar{\rho}_{0}$.

We define $\phi(u)$ on $\bar{\lambda}$ and $\gamma(v)$ on $\bar{\rho}$ by $\phi=f(x(u, 0))$ and $\gamma=$ $g(x(1, v))$. Since $x(u, 0)$ is bounded and increasing on $\bar{\lambda}, \phi$ is a function of bounded variation on $\bar{\lambda}$. Similarly, $\gamma$ is a function of bounded variation on $\bar{\rho}$.

Let $S_{w}$ be the subset of $\bar{R}_{w}$ in which $u<1$ and $v>0$. We define a new potential problem $P_{w}$ : find a function $\tau(u, v)$ harmonic in $S_{w}$, bounded in $\bar{R}_{w}$, and satisfying the boundary conditions

$$
\begin{gathered}
\tau_{u}=0 \text { on }\{u=0 ; v \in \rho\} \text { and } \tau=0 \text { on }\{u \in \lambda ; v=\kappa\}, \\
\lim \tau=\hat{\phi}(u) \text { as } v \longrightarrow+0 \text { for } u \in \lambda, \\
\lim \tau_{u}=-\hat{\gamma}(v)\left|z^{\prime}(w)\right| \text { as } u \longrightarrow 1-0 \text { for } v \in \rho .
\end{gathered}
$$

Since $z^{\prime}$ is continuous on $\bar{\rho}_{0}$ as a function of $v$ (Property C), it is an obvious verification to show $T$ is a solution to $P_{z}$ if, and only if, $\tau$ defined by $\tau(u, v)=T(x, y)$ is a solution to $P_{w}$.

We shall show that a solution to $P_{w}$ is

$$
\begin{aligned}
& \tau(u, v)=\sum_{n=1}^{\infty} \frac{g_{n}}{n} \sin \left(\frac{n \pi v}{\kappa}\right) \cosh \left(\frac{n \pi u}{\kappa}\right) \operatorname{csch}\left(\frac{n \pi}{\kappa}\right)+\frac{f_{0}}{2}\left(\frac{\kappa-v}{\kappa}\right) \\
& +\sum_{n=1}^{\infty} f_{n} \cos (n \pi u) \sinh (n \pi(\kappa-v)) \operatorname{csch}(n \pi \kappa) \text {, } \\
& g_{n}=\frac{-2}{\pi} \int_{0}^{\kappa} \gamma(v)\left[\left|\frac{d z}{d w}\right|\right]_{u=1} \sin \left(\frac{n \pi v}{\kappa}\right) d v, \quad n=1,2, \cdots, \\
& f_{n}=2 \int_{0}^{1} \phi(u) \cos (n \pi u) d u, \\
& n=0,1, \cdots
\end{aligned}
$$

Since $\phi$ and $\gamma\left|z^{\prime}\right|$ are functions of bounded variation, $f_{n}=O\left(n^{-1}\right)$ and $g_{n}=O\left(n^{-1}\right)$ [26, p. 48]. Thus $\tau$ is harmonic in $S_{w}$ and satisfies (9). By the Abel-Poisson sum theorem [26, p. 97], $\tau$ also satisfies 
the boundary conditions (10) and (11). It remains to show $\tau$ is bounded. The first series in (12) is dominated by a series with constant terms $O\left(n^{-2}\right)$ so that it is bounded on $\bar{R}_{w}$. If we set $\phi(-0)=$ $\phi(+0)$ and $\phi(1+0)=\phi(1-0)$, the second series in (12) tends to the bounded function $\hat{\phi}(u)$ as $v \rightarrow+0$ for $u \in \bar{\lambda}$. By the positiveness of summation A [26, p. 98], this series is bounded on $\bar{R}_{w}$. Thus $\tau$ is a solution to $P_{w}$.

We introduce $\eta(v)=\tau(1-0, v)$ for $v \in \bar{\rho}$. For future reference note that $\eta(v(x, 0))$ is in $L^{2}(\mathrm{x})$ because $\eta(v)$ is a bounded continuous function on $\rho$ and $v(x, 0)$ is continuous on $\bar{x}$. We introduce a third boundary value problem $Q_{w}$ : find a function $\nu(u, v)$ harmonic in $S_{w}$, bounded in $\bar{R}_{w}$, and satisfying the boundary conditions (9), (10), and $\nu \rightarrow \eta(v)$ as $u \rightarrow 1-0$ for $v \in \rho$. Clearly $\tau$ is a solution to $Q_{w}$. Let us show that it is the only solution. If $Q_{w}$ had more than one solution, there would exist a solution, say $\theta$, satisfying $Q_{w}$ with $\phi \equiv 0$ and $\eta \equiv 0$. Theorem W implies $\theta$ is continuous along $\lambda_{0}$ and $\rho_{0}$. Applying the maximum principle $[17$, p. 105] it follows that $\theta \equiv 0$ which shows $Q_{w}$ has at most one solution.

REMARK 3. The uniqueness of the solution to $Q_{w}$ is the key to the proof of Theorem 1, since from this uniqueness we obtain existence.

We proceed to our last boundary value problem $Q_{z}$ : find a function $U(x, y)$ harmonic in $S_{z}$, bounded in $\bar{R}_{z}$, satisfying (6), (7) and $U \rightarrow$ $\eta(v(x, 0))$ as $y \rightarrow+0$ for $x \in \mathfrak{r}$. Let $h(x)$ be defined by

$$
h(x)=f(x), x \in \overline{\mathfrak{l}} \text {, and } h(x)=\eta v(x, 0)), b<x \leqq \pi .
$$

Then $Q_{z}$ has a solution

$$
\begin{aligned}
U(x, y)= & \sum_{n=1}^{\infty}\left[j_{n} /(n-1 / 2)\right] \exp (-(n-1 / 2) y) \cos (n-1 / 2) x \\
& \frac{j_{n}}{n-1 / 2}=\frac{2}{\pi} \int_{0}^{\pi} h(x) \cos (n-1 / 2) x d x .
\end{aligned}
$$

Since $h \in L^{2}(\mathfrak{p})$, it follows after a bit of algebra that $\left\{j_{n}\right\}$ satisfies (5). Clearly, $U$ is a solution to $Q_{z}$ if, and only if, $\nu$ defined by $\nu(u, v)=$ $U(x, y)$ is a solution to $Q_{w}$. Since $\tau \equiv \nu, U$ is a solution to $P_{z}$ from which follows the validity of $(1 \mathrm{~A}-\mathrm{B})$.

We now examine uniqueness. If a second solution existed, there would be constants $\left(k_{1}, k_{2}, \ldots\right)$ satisfying (5) such that the function

$$
W(x, y)=\sum_{n=1}^{\infty}\left[\left(k_{n} /(n-1 / 2)\right] \cos (n-1 / 2) x \exp (-(n-1 / 2) y)\right.
$$

satisfies $P_{z}$, except perhaps for boundedness on $\bar{R}_{z}$, with $f$ and $g$ set equal to zero. To establish uniqueness it suffices (as will be seen) 
to show $W$ is continuous along $\overline{\mathfrak{p}}_{0}$. To this purpose we introduce the function $X$ conjugate to $W$,

$$
X(x, y)=C-\sum_{1}^{\infty}\left[k_{n} /(n-1 / 2)\right] \sin (n-1 / 2) x \exp (-(n-1 / 2) y),
$$

where $C$ is a constant. We set $F(z)=X+i W$ and $G=F_{y}$ so that

$$
G(z)=-i \sum_{1}^{\infty} k_{n} \exp (i(n-1 / 2) z) .
$$

Let us now show that $G=o\left(y^{-3 / 2}\right)$ as $y \rightarrow+0$ uniformly in $x$ for $x \in \overline{\mathfrak{p}}$. Indeed for $x \in \overline{\mathfrak{p}}$ and $y \leqq 2$, one finds $|G|$ is bounded by $e \sum\left|k_{n}\right| r^{n}$ where $r=e^{-y}$. Now by virtue of (5) there is a sequence of nonnegative constants $\left\{\delta_{n}\right\}$ such that $\delta_{n}=o(1)$ and

$$
\frac{\sum_{1}^{\infty}\left|k_{n}\right| r^{n}}{1-r}=\sum_{n=1}^{\infty}\left[\sum_{m=1}^{n}\left|k_{m}\right|\right] r^{n} \leqq \sum_{n=1}^{\infty} \delta_{n} n^{3 / 2} r^{n}=o\left([1-r]^{-5 / 2}\right) .
$$

This establishes the required growth for $G$. Since $W_{y} \rightarrow 0$ as $y \rightarrow+$ 0 for $x \in \mathfrak{x}$, it follows from Theorem $\mathrm{W}$ that $G$ is analytic on $\mathfrak{x}_{0}$ so that $X$ is constant on $\mathfrak{x}_{0}$. Choose $C$ such that $X=0$ on $\mathfrak{x}_{0}$. Let $H=$ $i(z-b)^{1 / 2} F$, and write $H=H_{1}+i H_{2}$. Clearly, $H$ is analytic in $R_{z}$ and $H_{2}(x, y) \rightarrow 0$ as $y \rightarrow+0$ for $x \in \mathfrak{I} \cup \mathfrak{x}$. By modifying the argument used above for $G$ it follows from (5) that $F=o\left(y^{-1 / 2}\right)$ as $y \rightarrow+0$ uniformly in $x$ for $x \in \bar{p}$. This implies: $H_{2}(b, y) \rightarrow 0$ as $y \rightarrow+0 ; H=$ $o\left(y^{-1 / 2}\right)$ uniformly in $x$ for $x \in \bar{p}$. Whence by Theorem $\mathrm{W}, H$ is analy tic on $\mathfrak{p}_{0}$. Therefore $W$ is continuous on $\mathfrak{p}_{0}$. Since $W$ can be reflected through $\{x=0 ; y>0\}$ and $\{x=\pi ; y>0\}$ similar, but easier, arguments show $W$ is continuous at $z=0$ and $z=\pi$. Applying the maximum principle $[17$, p. 75$]$ we obtain $W \equiv 0$. Since $\{\cos (n-1 / 2) x\}$ is a complete orthogonal set, $k_{n}=0$ for $n=1,2, \cdots$.

3.0. Behavior of the expansions. We describe certain characteristics of the solutions and the ways in which our results can be considered best possible. Let us denote by $S_{i}(x)$ the series in eq. (iA), e.g.,

$$
S_{1}(x)=\sum_{1}^{\infty}\left[j_{n} /(n-1 / 2)\right] \cos (n-1 / 2) x
$$

THEOREM 2. Let us assume the hypothesis of Theorem 1. Then $S_{i}(x), i=1,2,3,4$, converges to an absolutely continuous function on $\overline{\mathfrak{x}}$, has two sided continuity at $x=b$, in particular,

$$
S_{i}(b)=f(b-0) \text {; }
$$


the coefficients $\left(j_{1}, j_{2}, \ldots\right)$ satisfy

$$
j_{n}=O(1) \text {; }
$$

summation $A$ in eq. (iA) can be replaced by ordinary summation.

Proof. From Properties A and B of the mapping function, we see that $v(x, 0)$ is absolutely continuous on $\overline{\mathfrak{x}}$. Now let us show that $\eta(v)$ is absolutely continuous on $\bar{\rho}$. First we consider the series

$$
I(v)=\frac{\pi}{\kappa} \sum_{n=1}^{\infty} g_{n} \operatorname{coth}\left(\frac{n \pi}{\kappa}\right) \cos \left(\frac{n \pi v}{\kappa}\right), v \in \bar{\rho} .
$$

Since $g_{n}=O\left(n^{-1}\right)$, it follows that $I \in L^{2}(\rho)$ and the series can be integrated term by term [26, p. 59]. The resulting function, $J=\int_{0}^{v} I d v$, is absolutely continuous and $J(0)=0$. Next we consider the series

$$
F(v)=f_{0}[(\kappa-v) / 2 \kappa]+\sum_{n=1}^{\infty}(-1)^{n} f_{n} \sinh (n \pi(\kappa-v)) \operatorname{csch}(n \pi \kappa),
$$

Clearly, $\eta=F+J$. Since $f_{n}=O\left(n^{-1}\right), F$ is an infinitely differentiable function on $\delta \leqq v \leqq \kappa$ for $\delta>0$. After a little algebra one can write

$$
F(v)=\frac{f_{0}}{2}+\sum_{n=1}^{\infty}(-1)^{n} f_{n} e^{-n \pi v}-\frac{f_{0} v}{2 \kappa}+\sum_{n=1}^{\infty} f_{n} p_{n}(v)
$$

where $p_{n}^{(m)}=O\left(e^{-n}\right), m=0,1$, uniformly in $v$ for $v \in \bar{\rho}$, and further $p_{n}(0)=0$. Therefore utilizing the fact that $\cos n \pi u$ is symmetric about $u=1$ and the Abel-Poisson summation theorem [26, p. 97] one concludes that $F(+0)=f(b-0)$ and $F$ is continuous on $\bar{\rho}$. Let $\Phi(u)$ denote the even extension of $\phi$ with period 2 so that

$$
f_{0} / 2+\sum_{n=1}^{\infty} f_{n} \cos (n \pi u)=\hat{\Phi}(u), \quad-\infty<u<\infty .
$$

At the point $u=1, \hat{\Phi}$ has a symmetrical derivative equal to zero so that Fatou's theorem [4, p. 160] implies

$$
\lim \sum_{n=1}^{\infty}\left((-1)^{n} f_{n} n\right) r^{n}=0 \text { as } r \longrightarrow 1-0 .
$$

Thus $F^{\prime}(v)$ is continuous on $\bar{\rho}$. Consequently, $\eta(v)$ is absolutely continuous on $\bar{\rho}$. Since $v(x, 0)$ is increasing and absolutely continuous on $\overline{\mathfrak{r}}, \eta(v(x, 0))$ is absolutely continuous on $\overline{\mathfrak{x}}$ as a function of $x[15$, p. 195]. From the computations above, $h$ has two sided continuity at $x=b$, so that by the Dirichlet-Jordan theorem (18) is valid as well as the convergence of $S_{1}$ on $\overline{\mathfrak{x}}$. Since $h$ is of bounded variation on $\overline{\mathfrak{p}}$, (19) follows from (17). Finally, by Littlewood's Tauberian theorem [26, p. 81] we can replace summation $A$ in (1A) by ordinary summation. 
We turn our attention to examining the extent to which the results in Theorems 1 and 2 can be regarded as best possible.

Under the hypothesis of Theorem 1 , we know that $h$ is of bounded variation on $\bar{p}$. Therefore, if $j_{n}=o(1)$, it follows from (17) that $h$ is continuous on $\mathfrak{p}[26, \mathrm{p} .60]$, which is false in general. If $j_{n} \neq o(1)$, then by the Cantor-Lebesgue theorem [4, p. 174] the series in (iB) do not converge in the ordinary sense on any set of positive measure.

Let us consider eq. (2A-B) with $b=1, f \equiv 1$, and $g \equiv 0$. For this case an easy computation shows

$$
j_{n}=\frac{2}{\pi}+\frac{2}{\pi} \int_{1}^{\pi} h^{\prime}(x) \cos (n-1 / 2) x d x .
$$

Since $h$ is absolutely continuous on $\overline{\mathfrak{x}}$, it follows that $h^{\prime} \in L(\mathfrak{x})$ $[15$, p. 268]. Therefore, by the Riemann-Lebesgue theorem [26, p. 45] the integral in (20) tends to zero as $n \rightarrow \infty$. Hence, the estimate (19) cannot be improved.

Finally, we show that (5) is a best possible estimate for uniqueness, i.e., if (5) is replaced by

$$
\sum_{1}^{N}\left|j_{n}\right|=O\left(N^{3 / 2}\right)
$$

uniqueness fails. Counterexamples can be constructed for each of the four dual equations. The simplest example we have found is for eq. (2A-B). Consider the function

$$
T(x, y)=\left\{\frac{\left(\cos ^{2} x+\sinh ^{2} y\right)^{1 / 2}-\cos x \cosh y}{2\left(\cos ^{2} x+\sinh ^{2} y\right)}\right\}^{1 / 2},(x, y) \in R_{z},
$$

where the square roots are taken nonnegative. It is easy to verify that $T$ is harmonic in $S_{z}$ and satisfies the boundary conditions $T=0$ on $\{x=0 ; y>0\}, T_{x}=0$ on $\{x=\pi ; y>0\}$,

$$
\lim T=0 \text { for } x \in \mathfrak{I} \text { and } \lim T_{y}=0 \text { for } x \in \mathfrak{x} \text { as } y \longrightarrow+0
$$

with $b=\pi / 2$. It is also clear that

$$
\lim T=(-\cos x)^{-1 / 2} \text { as } y \longrightarrow+0 \text { for } x \in \mathfrak{x} .
$$

After verifying in the fashion of $\S 2$ that an appropriate uniqueness theorem holds, it follows that $T$ has the representation

$$
\begin{gathered}
T(x, y)=\sum_{1}^{\infty}\left[j_{n} /(n-1 / 2)\right] \sin (n-1 / 2) x \exp (-(n-1 / 2) y) \\
\frac{j_{n}}{n-1 / 2}=\frac{2}{\pi} \int_{\pi / 2}^{\pi} \frac{\sin (n-1 / 2) x}{(-\cos x)^{1 / 2}} d x
\end{gathered}
$$


If we set $t=x-\pi / 2$ and use the addition formula for sines, we find that $j_{n} /(n-1 / 2)$ is the sum of four integrals with constant multipliers whose absolute values are independent of $n$. One of these integrals is

$$
I=\int_{0}^{\pi / 2} \frac{\sin n t \cos (t / 2)}{(\sin t)^{1 / 2}} d t
$$

Now

$$
\frac{1}{\sin t}=\frac{1+t^{2} G(t)}{t} \text { and } \cos \frac{t}{2}=1+L(t), 0 \leqq t \leqq \frac{\pi}{2}
$$

where $G$ and $L$ are analytic. Thus

$$
I=\int_{0}^{\pi / 2} \frac{\sin n t}{\sqrt{t}} d t+O\left(n^{-1}\right)
$$

Further, if we set $\alpha_{n}=\sqrt{(n \pi / 2)}$, then

$$
\int_{0}^{\pi / 2} \frac{\sin n t}{\sqrt{t}} d t=\frac{2}{\sqrt{n}} \int_{0}^{\alpha_{n}} \sin \xi^{2} d \xi=O\left(n^{-1 / 2}\right)
$$

because of well known properties of Fresnel integrals. The three remaining integrals in the sum for $j_{n} /(n-1 / 2)$ can similarly be shown to be $O\left(n^{-1 / 2}\right)$. Thus $\left\{j_{n}\right\}$ satisfies $(21)$ and is a nonzero solution to eq. $(2 \mathrm{~A}-\mathrm{B})$ with $f \equiv 0$ and $g \equiv 0$. In summary we have established

THEOREM 3. Theorems 1 and 2 are sharp in the following sense. Theorem 1 is false if summation $A$ in (iB) is replaced for any $i$, $i=1,2,3,4$, by ordinary summation on any subset of $\mathfrak{x}$ of positive measure. The growth estimate (5) is best possible for if (5) is replaced by (21) the uniqueness assertion in Theorem 1 is false. The estimate (19) is best possible for if (19) is replaced by $j_{n}=o(1)$, then Theorem 2 is false.

\section{REFERENCES}

1. A. A. Bablojan and N. O. Gulkanjan, $A$ certain mixed problem for a rectangle (Russian), Akad. Nauk Armjan. SSR. Ser. Meh., 22 (1969), no. 1, 3-16.

2. A. A. Bablojan and V. G. Saakyan, On a plane contact problem in the theory of elasticity for a circular ring (Russian), Izv. Akad. Nauk Armjan. SSR Ser. Meh., 23 (1970), 3-17.

3. A. A. Bablojan, The solution of some dual series (Russian), Akad. Nauk Armjan, SSR Dokl., 39 (1964), 149-157.

4. N. K. Bary, A Treatise on Trigonometric Series, Vol. I, (translated from the Russian), MacMillan, New York, 1964.

5. B. P. Belinskii, Fourier series and integrals related to dual equations (Russian), Zap. Naucn. Sem. Leningrad Otdel. Mat. Inst. Steklov. (LOMI) 15 (1969), 66-84. 
6. M. Bentwich, Solution of Levi-Civita's problem by infinite matrix inversion, Trans. ASME Ser. E. J. Appl. Mech., 40 (1973), 31-36.

7. S. C. Das, On Tranter's method of solution of dual trigonometric sine series, J. Tech. Bengal. Engrg. College, 12 (1967), 75-90.

8. A. G. Ishakova, On the bending of a circular plate and an infinite strip lying on an elastic half-space (Russian). Izv. Akad. Nauk SSSR, Otedeleniye teh. nauk, no. 10 (1958), 87-91.

9. R. B. Kelman and C. A. Koper Jr., Separated variables solution for steady temperatures in rectangles, Trans. ASME Ser. C, J. Heat Transfer, 95 (1973), 130-132.

10. R. B. Kelman and C. A. Koper Jr., Least squares approximations for dual trigonometric series, Glasgow Math. J., 14 (1973), 111-119.

11. R. B. Kelman and R. P. Feinerman, Dual orthogonal series, SIAM J. Math. Anal., 5 (1974), 489-502.

12. T. Kiyono, and M. Shimasaki, On the solution of Laplace's equation by certain dual series equations, SIAM J. Appl. Math., 21 (1971), 245-257.

13. M. A. Lawrentjew and B. W. Schabat, Methoden der komplexen Funktionentheorie, VED Deutscher Verlag. Berlin, 1967.

14. W. Magnus, Ueber einige beschrankte Matrizen, Arch. Math., 2 (1949/50), 405-412.

15. M. E. Munroe, M. E. Introduction to Measure and Integral, Addison-Wesley, Cambridge, Mass., 1953.

16. B. Noble and M. Hussain, Angle of contact for smooth elastic inclusions, appearing in Developments in mechanics, IV (1967), 459-476, J. E. Cermak, and J. R. Goodman eds. 17. M. H. Protter and H. F. Weinberger, Maximum Principles in Differential Equations, Prentice-Hall, Englewood Cliffs, N. J., 1967.

18. W. M. Shepherd, On trigonometrical series with mixed conditions, Proc. London Math. Soc., Ser. 2, 43 (1937), 366-375.

19. I. N. Sneddon, Mixed Boundary Value Problems in Potential Theory, NorthHolland Pub. Co., Amsterdam, 1966.

20. I. N. Sneddon and M. Lowengrub, Crack Problems in the Classical Theory of Elasticity, Wiley, New York, 1969.

21. R. P. Srivastav, Dual series relations V, A generalized Schlomlich series and the uniqueness of the solution of dual equations involving trigonometric series, Proc. Roy. Soc. Edinburgh, Sec. A, 66 (1962/64), 173-184.

22. W. F. Trench and P. A. Scheinok, On the inversion of a Hilbert type matrix, SIAM Rev., 8 (1966), 57-61.

23. A. I. Tseitlin, On the methods of dual integral equations and dual series and their applications to the problems of mechanics (Russian), Prikl. Mat. Meh., 30 (1966), 259-270.

24. W. E. Williams, A note on integral equations, Glasgow Math. J., 13 (1972), 119121.

25. F. Wolf, Extensions of analytic functions, Duke Math. J., 14 (1947), 877-887.

26. A. Zygmund, Trigonometric Series, Vol. I, 2nd ed., Cambridge University Press, 1968.

Received August 12, 1973 and in revised form May 6, 1974. It is noted with appreciation that this paper was written while the author was a guest of the University of California at Berkeley. Professor Henry Helson is thanked for his admonitions which eliminated needless and tedious calculations. I am grateful to Professor Frantisek Wolf for his insightful suggestions, in particular, pointing out the relevance of the reflection principle in [25] which allowed the proofs to be shortened and the conclusions to be sharpened. This work was supported in part by the U. S. Army Research Office under grant No. DAH CO4 74G0140. 



\section{PACIFIC JOURNAL OF MATHEMATICS}

\section{EDITORS}

RICHARD ARENS (Managing Editor) University of California

Los Angeles, California 90024

\section{R. A. Beaumont}

University of Washington Seattle, Washington 98105
J. DugundJI Department of Mathematics University of Southern Californıa Los Angeles, California 90007

D. Gilbarg aNd J. Milgram Stanford University Stanford, California 94305

\section{ASSOCIATE EDITORS}

E. F. BeCKENBACH

B. H. NeUmanN

F. Wolf

K. YoSHIDA

\section{SUPPORTING INSTITUTIONS}

UNIVERSITY OF BRITISH COLUMBIA CALIFORNIA INSTITUTE OF TECHNOLOGY UNIVERSITY OF CALIFORNIA MONTANA STATE UNIVERSITY UNIVERSITY OF NEVADA NEW MEXICO STATE UNIVERSITY OREGON STATE UNIVERSITY UNIVERSITY OF OREGON OSAKA UNIVERSITY
UNIVERSITY OF SOUTHERN CALIFORNIA STANFORD UNIVERSITY UNIVERSITY OF TOKYO UNIVERSITY OF UTAH WASHINGTON STATE UNIVERSITY UNIVERSITY OF WASHINGTON AMERICAN MATHEMATICAL SOCIETY NAVAL WEAPONS CENTER 


\section{Pacific Journal of Mathematics \\ Vol. 59, No. 1 \\ May, 1975}

Shashi Prabha Arya and M. K. Singal, More sum theorems for topological

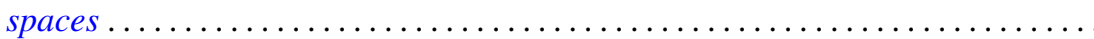

Goro Azumaya, F. Mbuntum and Kalathoor Varadarajan, On M-projective and

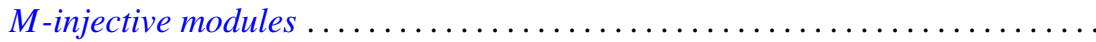

Kong Ming Chong, Spectral inequalities involving the infima and suprema of

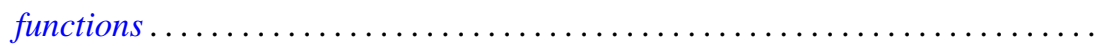

Alan Hetherington Durfee, The characteristic polynomial of the monodromy .......

Emilio Gagliardo and Clifford Alfons Kottman, Fixed points for orientation

preserving homeomorphisms of the plane which interchange two points ......

Raymond F. Gittings, Finite-to-one open maps of generalized metric spaces .......

Andrew M. W. Glass, W. Charles (Wilbur) Holland Jr. and Stephen H. McCleary,

$a^{*}$-closures of completely distributive lattice-ordered groups .............

Matthew Gould, Endomorphism and automorphism structure of direct squares of

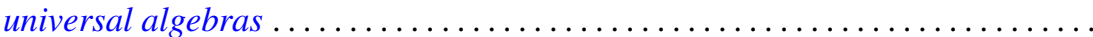

R. E. Harrell and Les Andrew Karlovitz, On tree structures in Banach spaces .....

Julien O. Hennefeld, Finding a maximal subalgebra on which the two Arens

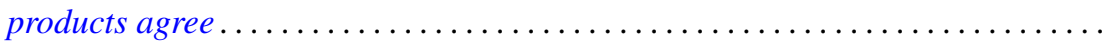

William Francis Keigher, Adjunctions and comonads in differential algebra .......

Robert Bernard Kelman, A Dirichlet-Jordan theorem for dual trigonometric

series

Allan Morton Krall, Stieltjes differential-boundary operators. III. Multivalued operators-linear relations...

Hui-Hsiung Kuo, On Gross differentiation on Banach spaces .

Tom Louton, A theorem on simultaneous observability ...

Kenneth Mandelberg, Amitsur cohomology for certain extensions of rings of algebraic integers.

Coy Lewis May, Automorphisms of compact Klein surfaces with boundary . . .

Peter A. McCoy, Generalized axisymmetric elliptic functions .

211

Muril Lynn Robertson, Concerning Siu's method for solving $y^{\prime}(t)=F(t$, $y(g(t)))$. .

Richard Lewis Roth, On restricting irreducible characters to normal

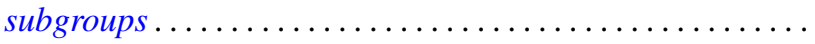

Albert Oscar Shar, $P$-primary decomposition of maps into an $H$-space .

Kenneth Barry Stolarsky, The sum of the distances to certain pointsets on the unit circle.

Bert Alan Taylor, Components of zero sets of analytic functions in $C^{2}$ in the unit ball or polydisc

Michel Valadier, Convex integrands on Souslin locally convex spaces ...

Januario Varela, Fields of automorphisms and derivations of $C$

Arnold Lewis Villone, A class of symmetric differential operators with deficiency

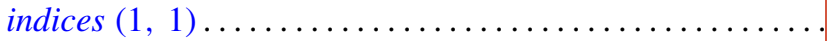

